\author{
C.-P. Heidegger \\ J.-A. Romand \\ M. M. Treggiari \\ C. Pichard
}

\section{Reply to the comments by Drs. Marik and Emery and Dr. Singer}

Accepted: 4 July 2007

Published online: 1 August 2007

(C) Springer-Verlag 2007

This reply refers to the comments available at: http://dx.doi.org/10.1007/ s00134-007-0805-x and http://dx.doi.org/ 10.1007/s00134-007-0806-9.

Sir: We would to like to thank Dr. Singer for his correspondence. The point is extremely well taken, and we share the view that accurately measuring metabolic expenditures is an important goal. We want to emphasize the lack of randomized controlled trials demonstrating the effectiveness of this approach in the critical care setting. Although it is true that the caloric targets may be inaccurate based on prediction formulas instead of measurement with calorimetry, it is very common in critically ill patients to lag behind for several days before achieving an adequate caloric intake, and in most cases a caloric deficit develops. Therefore even if the caloric goal is not established precisely, the risk of underfeeding is particularly high at least in the first few days after admission to the ICU. With the use of supplemental nutrition particular attention should be paid to prevent excessive caloric intake.

We also thank Drs. Marik and Emery for their commentary. It seems that in fact their views and ours are very close. As the authors mention, the Canadian guidelines recommend to maximize the EN before administrating $\mathrm{PN}$, and this is exactly what we propose in our clinical commentary (see Fig. 1). Indeed, we recommend that supplemental PN be considered only if protein and calorie targets are not reached by the enteral route at day 4. This proposal is also in line with the recently published ESPEN guidelines (Clinical Nutrition, 2006).
The various studies cited in favor of "permissive underfeeding" are definitively not in line with the recommendations mentioned above. Many studies have demonstrated that the ICU patient population is at greater risk of underfeeding than "eufeeding", and in our opinion targeting prolonged underfeeding must not be a recommendation in this population. Finally, and this is probably the most important point, we have also mentioned that a well conducted prospective clinical trial is needed to confirm this approach of combined nutrition.

\author{
C.-P. Heidegger ( $)$ J J.-A. Romand \\ M. M. Treggiari \\ Geneva University Hospital, Service of \\ Intensive Care, \\ Rue Micheli-du-Crest 24, 1211 Geneva 14, \\ Switzerland \\ e-mail: claudia-paula.heidegger@hcuge.ch \\ Tel.: +41-22-3827460 \\ Fax: +41-22-3827470 \\ C. Pichard \\ Geneva University Hospital, Clinical \\ Nutrition, \\ 1211 Geneva 14, Switzerland
}

